\title{
Synthesis of Nanometer-sized Rod-Coil Block Copolymers
}

\author{
Hannah M. König and Andreas F. M. Kilbinger*
}

\begin{abstract}
We present the solid-supported synthesis of a linear rigid rod-like tetradeca(p-benzamide) nanorod as well as a pentadeca(p-benzamide) nanorod carrying an amide $N$-hexyl side chain at the center of the rod (on the $8^{\text {th }}$ amino acid of the molecule). These nanorods were conjugated with solubilizing poly(ethylene glycol) chains and their solution aggregation was investigated. Both rod-coil block copolymers form soluble aggregates even in polar aprotic solvents such as DMF and DMSO. We show that sequence-controlled nanometer-sized shape persistent aramide scaffolds can readily be prepared using solid-supported synthesis.
\end{abstract}

Keywords: Aramide · Block copolymers · Oligomers · Self-assembly · Solid-supported synthesis

Shape-persistent materials, in particular molecular rods with dimensions on the nanoscale are envisaged to be important scaffold materials for bottom-up approaches to nanomaterials. ${ }^{[1,2]}$ Moreover, rod-like materials that are truly monodisperse and can be sequentially built with monomer sequence control are very attractive in this context. ${ }^{[3-17]}$ Such materials would not only allow the shape-persistent construction of larger nanostructures but also allow the placement of functional groups at pre-determined positions within the rigid scaffold. Several synthetic strategies to rod-like molecules based on aramides have been described by us in the past: manual ${ }^{[18,19]}$ and automated ${ }^{[20]}$ solution syntheses as well as manual[ ${ }^{[3]}$ and automated ${ }^{[21]}$ solid-supported syntheses have been reported. For rod-coil copolymers based on well-defined oligo( $p$-benzamide)s (OPBA) and polydisperse poly(ethylene glycol) (PEG) we found that an oligomer length of seven repeat units was sufficient to induce strong aggregation in non-polar organic solvents such as chloroform or toluene. ${ }^{[4]}$ A molecular mechanics model of an OPBA heptamer shows that the energetically most stable all-trans amide form has a C-to-N-terminal length of $c a .4 .4 \mathrm{~nm}$. In order to access larger nanostructures longer scaffolds than an OPBA heptamer would need to be synthetically accessible.

${ }^{*}$ Correspondence: Prof. A. F. M. Kilbinger

Department of Chemistry

University of Fribourg

Chemin du Musée 9

$\mathrm{CH}-1700$ Fribourg

E-mail: andreas.kilbinger@unifr.ch
Here we describe the solid-supported synthesis of an OPBA tetradecamer and a pentadecamer as well as their conjugation with poly(ethylene glycol) and investigation of block copolymer aggregation via transmission electron microscopy (TEM).

The solid-supported OPBA tetradecamer 1a was synthesized on Wang resin $\left(0.6 \mathrm{mmol} \mathrm{g}^{-1}\right)$ using the acid chloride of $N$-Fmoc (9-fluorenylmethyl chloroformate) and $N$-PMB ( $p$-methoxybenzyl) protected 4-aminobenzoic acid according to a reported procedure. ${ }^{[4]}$ Commercially available Wang resin was pre-functionalized with $N$-Fmoc-4-aminobenzoic acid which resulted in oligomers that did not carry the PMB protective group on the first phenyl ring (closest to the C-terminus, see Figs 1 and 2, top). The solid-supported synthesis of the tetradecamer 1a (Fig. 1, top) was achieved in $68 \%$ overall yield over 14 amide coupling steps and 14 Fmoc deprotection steps (see Fmoc-cleavage elugram, Fig. 1, bottom). This corresponds to a yield of $97 \%$ per amide coupling. As shown pre-

viously[3,22-25] $\quad N$-alkylated benzanilides adopt a cis-conformation $(E)$ with respect to the phenyl rings leading to a coil-like oligomer chain. After acidic deprotection of the $N$-PMB groups the tetradecamer 1a shown in Fig. 1, top, (shown as a linear trans-conformer for clarity) will adopt a straight linear rigid rod-like conformation (trans, $Z$ ). We have previously shown that copolymers of these rod-like oligomers form $\beta$-sheet-like aggregates in solution. $[4,26,27]$

The terminal amine of solid supported oligomer 1a was further reacted with 4-pentynoic acid chloride to place a terminal alkyne on the $N$-terminus of $\mathbf{1 a}$. Poly(ethylene glycol)monomethyl ether mono azide ( $\left.\mathrm{Mn} c a .5000 \mathrm{~g} \mathrm{~mol}^{-1}\right)^{[4]}$ was subsequently reacted with the terminal alkyne of 1a in analogy to a reported procedure. ${ }^{[4]}$ Cleavage from the resin yielded the PEG-OPBA conjugate in its $N$-PMB protected form. This rod-coil precursor polymer (1b, see Scheme 1) is soluble in common organic solvents such as chloroform,

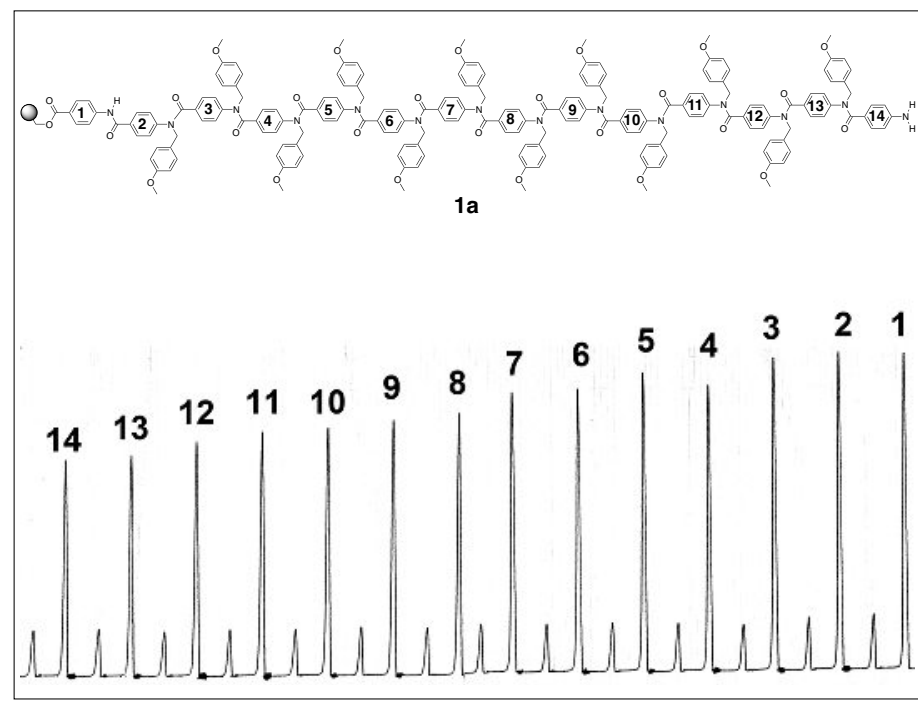

Fig. 1. Top: Chemical structure of solid supported tetradecamer 1a. All but the first amide carry an acid labile $p$-methoxybenzyl protective group. Bottom: Fmoccleavage elugram quantifying the successful synthesis over altogether 28 synthetic steps (including amide coupling and Fmoc deprotection) in $68 \%$ overall yield. The numbering refers to the number of the amino acid coupled (see top). 
THF or DMSO and shows no aggregation when analyzed by chloroform gel permeation chromatography (GPC, see Fig. 3).

Solid-supported $p$-aminobenzoic acid oligomer 2a (Fig. 2, top) was prepared in an analogous manner to 1a. ${ }^{[3,4]}$ However, the eighth aromatic amino acid (counted from the $C$-terminus) carried an $N$-hexyl side chain, which is stable under the acidic cleavage conditions for the PMB protective group (TFA:DCM = 1:1 vol/ vol). As tertiary aromatic amides adopt a cis-conformation $(E)$ with respect to the phenyl rings (vide supra), the eighth amide (counted from the $C$-terminus) should adopt a cis conformation $(E)$ while all other amides should adopt trans-conformations $(Z)$. We speculated that this might lead to a V-shaped molecular rod, which should exhibit different aggregation phenomena compared to its linear rigid rod counterpart 1a.

Fig. 2, top, shows the molecular structure and the Fmoc-cleavage elugram (Fig. 2, bottom) for the $N$-protected 15 mer heterosequence 2a. In analogy to compound 1a, $\mathbf{2 a}$ was reacted with pentynoic acid chloride and subsequently PEG-monomethyl ether mono azide to yield the copolymer 2b. The $N$-protected precursor polymer $\mathbf{2 a}$ shows a similar organo-solubility as $\mathbf{1 a}$ and no aggregation can be observed by GPC analysis in chloroform (Fig. 3).

Both block copolymers $\mathbf{1 b}$ and $\mathbf{2 b}$ were purified by preparative GPC after acidic cleavage from the solid support. We attribute the noticeable shift in the analytical GPC elugrams (Fig. 3) between $\mathbf{1 b}$ and $\mathbf{2 b}$ to a fractionation during preparative GPC purification. ${ }^{1} \mathrm{H}-\mathrm{NMR}$ analysis of block copolymers $\mathbf{1 b}$ and $\mathbf{2 b}$ confirmed the successful attachment of the oligomers to PEG. As the solid-supported synthesis was carried out with $p$-nitrobenzoyl chloride capping steps after the reaction of every monomer unit, only the target structures shown in Figs 1 and 2 carried the reactive terminal alkynes for PEG-azide conjugation. In other words, all lower oligomers were non-functionally terminated and separated by preparative GPC. Unfortunately, MALDI-ToF mass spectrometry analysis of the copolymers $\mathbf{1 b}$ and $\mathbf{2 b}$ was not successful.

Copolymers $\mathbf{1 b}$ and $\mathbf{2 b}$ were dissolved in TFA $(100 \%)$ for $12 \mathrm{~h}$ at room temperature to cleave the PMB protective groups, as reported previously. ${ }^{[4]}$ The immediate formation of a dark red color upon addition of TFA to $\mathbf{1 b}$ and $\mathbf{2 b}$ indicated the successful PMB deprotection reaction. After 12 $\mathrm{h}$ the polymers $(\mathbf{1 c}, \mathbf{2 c})$ were precipitated into dichloromethane and re-dissolved in DMSO:toluene (vol:vol = 1:1) under sonication. After ca. $15 \mathrm{~min}$. a colorless solid of $1 \mathbf{c}$ and $2 \mathbf{c}$ started to precipitate from the solution. Optical microscopic investiga-

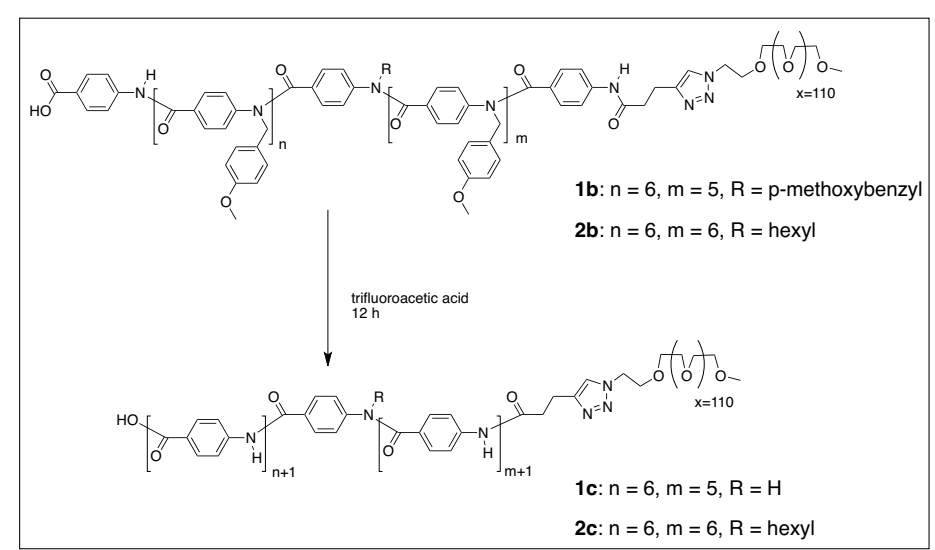

Scheme 1. N-Protected oligo( $p$-benzamide)-block-PEG copolymers $\mathbf{1 b}$ and 2b are $N$-deprotected under acidic conditions yielding nanorod-coil block copolymers 1c and $2 c$.

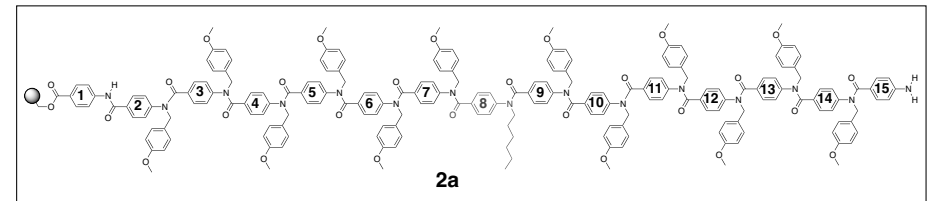

Fig. 2. Top: Chemical structure of solid supported pentadecamer 2a. All but the first and the eighth amide carry an acid labile p-methoxybenzyl protective group. The eighth amide carries a hexyl group which cannot be removed under acidic conditions. Bottom: Fmoc-

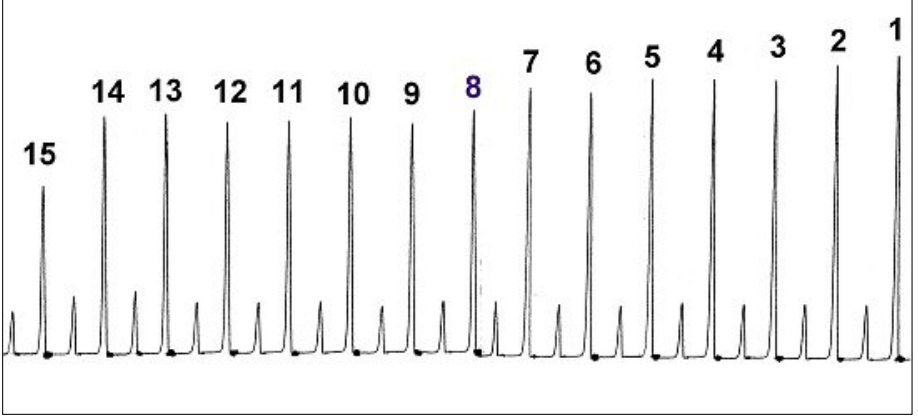
cleavage elugram quantifying the successful synthesis over altogether 30 synthetic steps (including amide coupling and Fmoc deprotection) in $54 \%$ overall yield. The numbering refers to the number of the amino acid coupled (see top).

tion of the solids under crossed polarizers indicated the presence of anisotropic order. To further investigate the aggregation of $\mathbf{1 c}$ and $\mathbf{2 c}$ we dropcast freshly prepared DMSO/toluene solutions (1c, vide supra) or chloroform solution (2c) for transmission electron microscopic (TEM) investigation.

As can be seen in representative TEM images (Fig. 4, top and bottom), long rigid

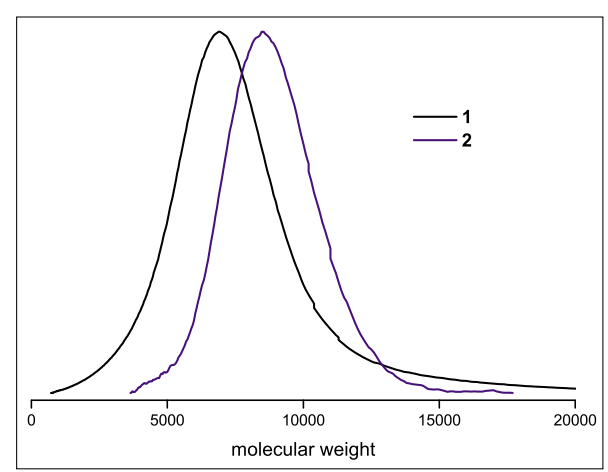

Fig. 3. GPC (chloroform) elugrams for $N$-protected OPBA-PEG block copolymers $1 \mathrm{~b}$ (black) and 2b (blue). rods are observed for both rod-coil block copolymers 1c and 2c. The molecular formation mechanism for such OPBA-PEG rod-coil block copolymers was previously reported ${ }^{[3,26,28]}$ and can be explained by a $\beta$-sheet-like aggregation of the OPBA blocks surrounded by a PEG corona. Within the rigid rod-like aggregates individual fibrils with widths of $c a .7 \mathrm{~nm}$ for both $\mathbf{1 c}$ and $2 \mathrm{c}$ can be seen. An observed width of $7 \mathrm{~nm}$ corresponds well to the width expected from a molecular mechanics model of the linear OPBA 14-mer 1c $(c a .9 \mathrm{~nm})$. However, this data does not support the assumption of a V-shaped geometry in case of block copolymer 2c which should exhibit a distinctly shorter width in the TEM projection. We assume that both $\mathbf{1 c}$ and 2c adopt the linear all-trans form upon aggregation. We hypothesize that the energetic penalty for an unfavored cis-trans ( $E$ to $Z$ ) conformational change is overcompensated by additional intermolecular hydrogen bond formation between neighboring linear OPBA rods. 


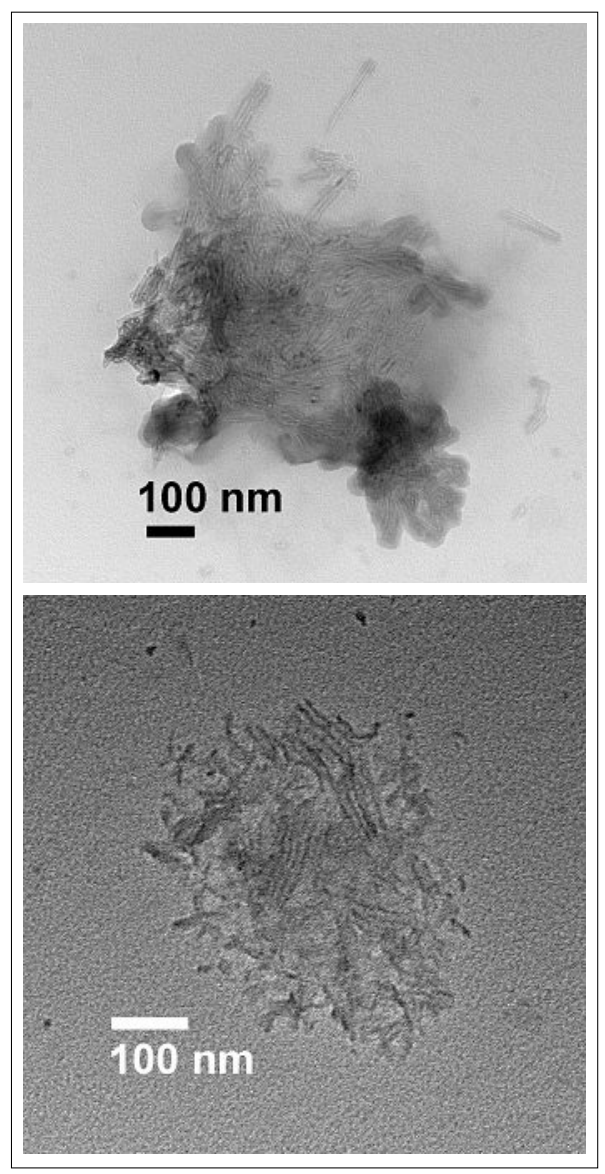

Fig. 4. Top: TEM image of OPBA-PEG block copolymer $1 \mathrm{c}$ deposited from DMSO solution (0.5 $\mathrm{mg} \mathrm{mL}^{-1}$ ) onto a carbon-coated nickel TEM grid. Bottom: TEM image of OPBA-PEG block copolymer $\mathbf{2 c}$ deposited from chloroform solution (0.5 $\left.\mathrm{mg} \mathrm{mL}^{-1}\right)$ onto a carbon-coated copper TEM grid.

\section{Conclusions}

The work described here shows that the fast automated solid-supported synthesis of long chain hydrophobic oligoaramides is possible up to the pentadecamer in good yields. This approach furthermore allows the sequence-specific introduction of derivatives of $p$-aminobenzoic acid, here, $\quad N$-hexyl- $N$-4'-methoxybenzyl-4aminobenzoic acid. Introduction of an $\mathrm{N}$-hexyl side chain at the central position (amino acid number eight counted from the C-terminus) of the pentadecamer was believed by us to alter the shape of the $N$-deprotected pentadecamer from alllinear to 'V-shaped' due to the cis (E) conformation of the $N$-hexylated amide. The pentadecamer and tetradecamer-PEG block copolymers precipitate from toluene/DMSO (1:1 vol:vol) solution upon $N$-deprotection. TEM investigation of the precipitate shows the formation of rodlike micelles. No evidence was found that the predicted non-linear geometry of the aramide oligomers existed in the solid state investigated by TEM. We believe that strong intermolecular hydrogen bond formation which is maximized in the linear oligomer overcompensates the energetic penalty for the cis $(E) N$-hexylated amide conformation.

\section{Experimental}

Solvents of technical grade were purchased from Acros Organics. Solvents of p.a. quality were purchased from Fisher Scientific. All other reagents were purchased from either Acros Organics or Sigma-Aldrich and used without further purification. Deuterated solvents were purchased from Deutero $\mathrm{GmbH}$. Dichloromethane was stored over calcium hydride and distilled freshly before use. NMP was provided by BASF SE Ludwigshafen, dried over $\mathrm{P}_{2} \mathrm{O}_{5}$ and freshly distilled prior to use.

${ }^{1} \mathrm{H}-\mathrm{NMR}$ spectra were recorded at 300 $\mathrm{MHz}$ on a Bruker AC 300 spectrometer and the spectra referenced with respect to residual protonated solvent. Gel-permeation chromatography elugrams (GPC) were recorded in chloroform with an apparatus consisting of a Waters 717 plus autosampler, a TSP Spectra Series P 100 pump and a set of three GPC separation columns (PSS SDV columns (104/500/50 Å)). Signals were recorded with a TSP Spectra System UV 2000 (UV at $254 \mathrm{~nm}$ ) and a Wyatt Optilab DSP (refractive index). The apparatus was calibrated with several polystyrene standards purchased from Polymer Standards Service (PSS).

Preparative GPC was carried out in DMF using a Knauer HPLC Pump 64, a Knauer Variable Wavelength Monitor (UV detector at $254 \mathrm{~nm}$ ) and an MZGPC $250 \times 30 \mathrm{~mm}$ SEC column (MZGel SDplus, 10E3 A, $10 \mu \mathrm{m}$ ) from MZAnalysentechnik, Mainz, Germany.

TEM measurements were recorded on a Philips EM 420 transmission electron microscope with $\mathrm{LaB}_{6}$ cathode and an accelerating voltage of $120 \mathrm{kV}$. TEMgrids (carbon film on copper or nickel, 300 mesh) were purchased from Electron Microscopy Sciences, Hatfield, PA, USA.

The automated synthesis of oligo $(p-$ benzamide)s was carried out on an Applied Biosystems model 431A peptide synthesizer with a modified synthesis protocol as described previously.[4]

Monomer and automated syntheses of shorter oligomers were reported previously. ${ }^{[4]}$

\section{Acknowledgement}

The authors thank BASF for generous donation of the solvent NMP and the Deutsche Forschungsgemeinschaft (DFG) and the Swiss National Science Foundation (SNF) for funding.

Received: July 5, 2013

[1] P. F. H. Schwab, M. D. Levin, J. Michl, Chem. Rev. 1999, 99, 1863.

[2] P. F. H. Schwab, J. R. Smith, J. Michl, Chem. Rev. 2005, 105, 1197.

[3] H. M. König, R. Abbel, D. Schollmeyer, A. F. M. Kilbinger, Org. Lett. 2006, 8, 1819.

[4] H. M. König, T. Gorelik, U. Kolb, A. F. M. Kilbinger, J. Am. Chem. Soc. 2007, 129, 704.

[5] H. Seyler, A. F. M. Kilbinger, Tet. Lett. 2013, 54,753 .

[6] C. M. Gothard, N. A. Rao, J. S. Nowick, J. Am. Chem. Soc. 2007, 129, 7272.

[7] J. C. Nelson, J. K. Young, J. S. Moore, J. Org. Chem. 1996, 61, 8160.

[8] J. K. Young, J. C. Nelson, J. S. Moore, J. Am. Chem. Soc. 1994, 116, 10841.

[9] S. Huang, J. M. Tour, J. Org. Chem. 1999, 64, 8898.

[10] S. Huang, J. M. Tour, J. Am. Chem. Soc. 1999, $121,4908$.

[11] L. Jones, J. S. Schumm, J. M. Tour, J. Org. Chem. 1997, 62, 1388.

[12] C. Chi, J. Wu, X. Wang, X. Zhao, J. Li, F. Wang, Macromolecules 2001, 34, 3812.

[13] P. R. L. Malenfant, J. M. J. Fréchet, Chem. Commun. 1998, 2657.

[14] C. G. Levins, C. E. Schafmeister, J. Am. Chem. Soc. 2003, 125, 4702 .

[15] E. E. Baird, P. B. Dervan, J. Am. Chem. Soc. 1996, 118,6141 .

[16] N. Wurtz, J. M. Turner, E. E. Baird, P. B. Dervan, Org. Lett. 2001, 3, 1201.

[17] V. Semetey, D. Moustakas, G. M. Whitesides, Angew. Chem., Int. Ed. 2006, 45, 588.

[18] R. Abbel, H. Frey, D. Schollmyer, A. F. M. Kilbinger, Chem. Eur. J. 2005, 11, 2170.

[19] R. Abbel, T. W. Schleuss, H. Frey, A. F. M. Kilbinger, Macromol. Chem. Phys. 2005, 206, 2067.

[20] J. Klos, F. Wurm, H. M. König, A. F. M. Kilbinger, Macromolecules 2007, 40, 7827.

[21] H. M. König, A. F. M. Kilbinger, Macromol. Rapid. Commun. 2008, 29, 1721.

[22] J. Clayden, L. Vallverdu, M. Helliwell, Org. Biomol. Chem. 2006, 4, 2106.

[23] A. Tanatani, A. Yokoyama, I. Azumaya, Y. Takakura, C. Mitsui, M. Shiro, M. Uchiyama, A. Muranaka, N. Kobayashi, T. Yokozawa, J. Am. Chem. Soc. 2005, 127, 8553.

[24] A. Itai, Y. Toriumi, S. Saito, H. Kagechika, K. Shudo, J. Am. Chem. Soc. 1992, 114, 10649.

[25] H. Masu, M. Sakai, K. Kishikawa, M. Yamamoto, K. Yamaguchi, S. Kohmoto, J. Org. Chem. 2005, 70, 1423.

[26] T. W. Schleuss, R. Abbel, M. Gross, D. Schollmeyer, H. Frey, M. Maskos, R. Berger, A. F. M. Kilbinger, Angew. Chem. Int. Ed. 2006, 45, 2969.

[27] H. Seyler, E. Berger-Nicoletti, A. F. M. Kilbinger, J. Mater. Chem. 2007, 17, 1954.

[28] A. Bohle, G. Brunklaus, M. R. Hansen, T. W. Schleuss, A. F. M. Kilbinger, J. Seltman, H. W. Spiess, Macromolecules 2010, 43, 4978. 